\title{
EAS Arrays at High Altitudes Start the Era of UHE $\gamma$-ray Astronomy
}

\author{
Zhen Cao ${ }^{1,2,3}$ (D)
}

1 Key Laboratory of Particle Astrophysics, Institute of High Energy Physics, Chinese Academy of Sciences, Beijing 100049, China; caozh@ihep.ac.cn

2 University of Chinese Academy of Sciences, Beijing 100049, China

3 Tianfu Cosmic Ray Research Center, Chengdu 610044, China

Citation: Cao, Z. EAS Arrays at High Altitudes Start the Era of UHE $\gamma$-ray Astronomy. Universe 2021, 7, 339. https://doi.org/10.3390/ universe7090339

Academic Editors: Ulisses Barres de Almeida and Michele Doro

Received: 10 August 2021

Accepted: 5 September 2021

Published: 9 September 2021

Publisher's Note: MDPI stays neutral with regard to jurisdictional claims in published maps and institutional affiliations.

Copyright: (c) 2021 by the authors. Licensee MDPI, Basel, Switzerland. This article is an open access article distributed under the terms and conditions of the Creative Commons Attribution (CC BY) license (https:/ / creativecommons.org/licenses/by/ $4.0 /)$.
Abstract: The evolution of extensive air shower detection as a technique for $\gamma$-ray astronomical instrumentation for the last three decades is reviewed. The first discoveries of galactic PeVatrons by the Large High Altitude Air Shower Observatory demonstrate the importance of this technique in ultra-high energy $\gamma$-ray astronomy. Utilizing this technique, the origins of high energy cosmic rays may be discovered in the near future.

Keywords: PeVatron; Crab Nebula; angular resolution; energy spectral distribution; $\gamma$-ray astronomy

\section{Introduction}

As of today, the Large High Altitude Air Shower Observatory (LHAASO) has detected a dozen PeVatrons. A few of them emit very energetic photons around and even above $1 \mathrm{PeV}$. It is a discovery that opens the window to Ultra High Energy (UHE, $\mathrm{E}_{\gamma}>0.1 \mathrm{PeV}$ ) $\gamma$-ray observation. The traditional cosmic ray (CR) detection method of observing extensive air shower (EAS) on the ground has evolved as a successful $\gamma$-ray detecting technique over three decades of remarkable development. The successful detection of PeVatrons in the UHE band indicates that both sensitivity and angular resolution of the EAS detection have reached the high standards of the $\gamma$-ray astronomical studies set by other very successful experimental research in Very High Energy $\left(\mathrm{VHE}, \mathrm{E}_{\gamma}>0.1 \mathrm{TeV}\right)$ and High Energy $(\mathrm{HE}$, $\mathrm{E}_{\gamma}>0.1 \mathrm{GeV}$ ) bands. The long-standing mystery of galactic CR origins may be uncovered in this new era of UHE CR observation utilizing EAS detection at high altitudes. As this technology may soon lead to further important discoveries, this paper reviews the history of EAS detection technology in $\gamma$-ray astronomy, outlining major achievements, advantages and disadvantages.

\section{The Pioneers of EAS Arrays for $\gamma$-ray Astronomy}

In the 1980s, at the onset of VHE $\gamma$-ray astronomy, the goal was to develop a technique that should be sensitive enough to detect $\gamma$-rays from a single source. However, it was unclear whether or not such a source existed, or more quantitatively what could have been the flux level of the $\gamma$-rays to be detected. It was a job of these pathfinders to create or improve detection techniques available at the time. EAS detection had already been a viable and acceptable technique for CRs at energies well above $100 \mathrm{TeV}$. A reasonable sampling rate in the shower fronts that allowed measurement of the shower direction and energy with desirable accuracies is economically acceptable. However, this technique suffered from well-known weaknesses. One of them was that the threshold energy was high at sea level where most arrays were at that time. The other was that the solution for distinguishing between CR and $\gamma$-ray events, thus suppressing CR background, was too expensive to be affordable. EAS technique therefore was not an obvious choice unless there existed a very strong motivation. The claim of $\gamma$-ray detection at PeV energy level [1] in 1983 provided the motivation required to spur a wave of experiments using EAS arrays. 
The working energies of a typical EAS array of shower particle counters with a typical spacing of about $15 \mathrm{~m}$ would be ideal for the detection of $\mathrm{PeV}$ events. Two experiments, CASA-MIA [2] in Utah and $\mathrm{AS}_{\gamma}$ [3] in Tibet, set out to overcome the shortcomings of the EAS detection method.

CASA-MIA [2], as schematically shown in Figure 1, was built to achieve a threshold energy around $0.1 \mathrm{PeV}$ by selecting the site at $1600 \mathrm{~m}$ above sea level (a.s.1.). Detectors spaced $15 \mathrm{~m}$ apart over $1 / 4 \mathrm{~km}^{2}$ attempted to observe the theorized very low flux of $\gamma$-ray photons. The array achieved an angular resolution of $1.2^{\circ}$ (hereafter in this paper, the angular resolution is defined as the space angle of a cone in which $68 \%$ of light from a point source is contained). In addition to the EAS particle counter array, a muon detector array with an active area of $2560 \mathrm{~m}^{2}$ was built to measure the $\mu$-content of EAS events. The $\mu$-content is much smaller in a $\gamma$-ray induced EAS than that by an ordinary CR particle; therefore, around $90 \%$ of the background CR events were rejected using the muon-poor cut method.
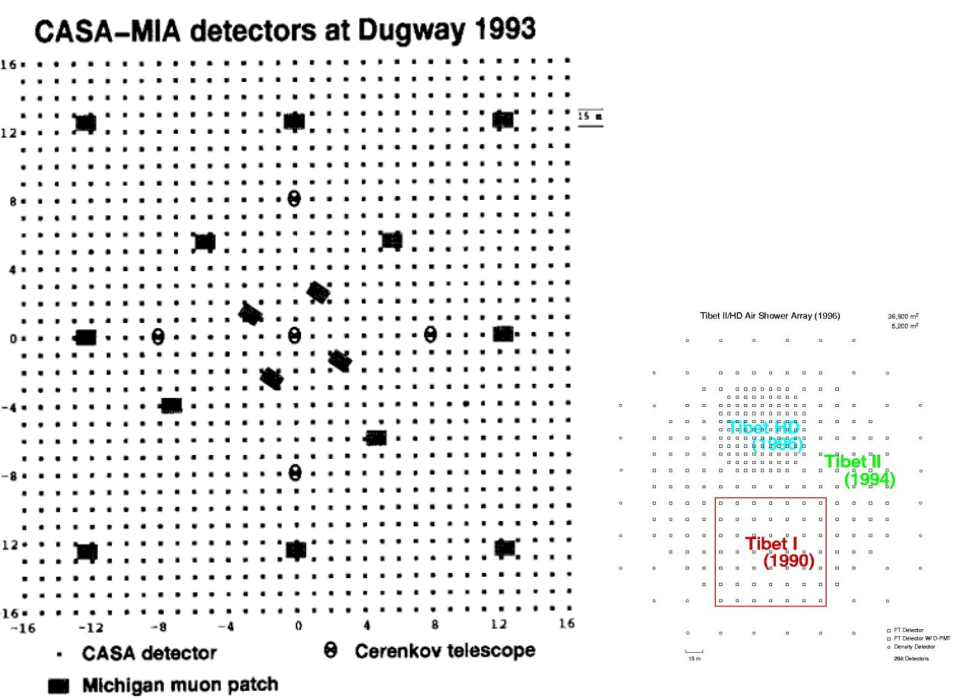

Figure 1. Schematic of layouts of the CASA-MIA array and $\mathrm{AS}_{\gamma}$ in phases before 2002.

$\mathrm{AS}_{\gamma}[3]$, schematically shown in Figure 1, is a smaller array compared with CASAMIA situated at $4300 \mathrm{~m}$ a.s.l. The array used smaller counters of $0.5 \mathrm{~m}^{2}$ with the identical spacing of $15 \mathrm{~m}$. Due to the high altitude, the threshold energy was below $10 \mathrm{TeV}$ and a similar angular resolution of $1^{\circ}$ was achieved. Without muon detector in the array, the detection of $\gamma$-rays from a point-like source was solely based on statistics. With a sufficient exposure to the source within the angular range defined by the Point Spread Function (PSF), the significance of $\sim N_{\gamma} / \sqrt{N_{C R}}$ eventually becomes acceptable, e.g., $>5 \sigma . N_{\gamma}$ is the number of photons from the source and the $N_{C R}$ is the number of background CR events estimated using the same size window as the PSF in a slightly different direction from the source. As both of them increase with the exposure time linearly, the significance of the detection of the $\gamma$-ray source will become statistically acceptable. Since the $\mathrm{AS}_{\gamma}$ experiment has had many major upgrades since 1993, the original array at that time has been referred to as Tibet-I. The difference between the two experiments, CASA-MIA and Tibet-I, was quite obvious.

\section{Altitudes of the EAS Array}

A few more years after the discovery of the first VHE $\gamma$-ray source, namely the Crab Nebula, referred to as the 'standard candle' in the northern sky nowadays, by Whipple experiment [4], the CASA-MIA collaboration published upper limits of $\gamma$-ray flux above $100 \mathrm{TeV}$ for Cyg X-3, Her X-1 [5] and the Crab Nebula [6]. In 1996, the angular resolution of the array had already been improved to be $0.5^{\circ}$ above $100 \mathrm{TeV}$. The experiment reached its limit and decided to terminate the operation. 
Remarkably, the $\mathrm{AS}_{\gamma}$ experiment was also able to set the limit of $\gamma$-ray flux for the Crab Nebula in 1992 [3] at much lower energy around $10 \mathrm{TeV}$. Without discrimination between $\gamma$-ray or proton induced showers, the small array (about 1/25 of CASA-MIA) of smaller scintillator counters ( $1 / 2$ of the size of counters in the CASA array) set the limit closer to the extrapolated flux from the Whipple experiment's measurements. This achievement highlighted the huge advantage of the high altitude of the array site. However, it is still difficult to reach to the sensitivity of $1 \mathrm{Crab}$ Unit (CU), i.e., the photon flux from the Crab Nebula. The experiment was upgraded to Tibet-III by 2002. To further take advantage of the site's high altitude, the spacing between counters was reduced down to $7.5 \mathrm{~m}$. This pushed the threshold energy lower but kept the same angular resolution of $1^{\circ}$. Lowering the threshold energy provided an advantage in signal-to-noise ratio $N_{\gamma} / \sqrt{N_{C R}}$ as long as the spectra of $\gamma$-rays from the sources were not too much harder than the CR spectrum. For the Crab Nebula, the spectral index is $\sim 2.6$, which is close enough to the CR's $\sim 2.7$.

Achieving the sensitivity of $1 \mathrm{CU}$ was certainly a landmark of the first generation of the VHE $\gamma$-ray EAS detectors. During the same period, two other techniques were also developed and brought some more diversities to the EAS detection community. They were water Cherenkov detector (WCD) array from the MILAGRO experiment and Resistive Plate Chamber (RPC) array from the ARGO-YBJ experiment.

\section{Background Suppression via $\gamma / C R$ Separation}

At Fenton Hill, New Mexico, US, the MILAGRO experiment [7] reused a water pool, $60 \mathrm{~m} \times 80 \mathrm{~m} \times 8 \mathrm{~m}$ as part of a Los Alamos geothermal experiment, at an altitude of $2600 \mathrm{~m}$ a.s.1. A $3 \mathrm{~m} \times 3 \mathrm{~m}$ grid of 8 " photo multiplier tubes (PMT) were deployed at the depth of $1.5 \mathrm{~m}$ beneath the surface to measure EAS secondary particles, mainly $\gamma$ photons, electrons and positrons $\left(e^{-}, e^{+}\right)$via Cherenkov light produced by sub-showers initiated by those EAS particles in water. In CR induced showers, certain amount of muons are produced and easily penetrate the entire depth of the pool. Muons have a wider lateral distribution than electromagnetic (EM) particles in a shower. At a distance far from the shower core, muons produce larger Cherenkov light signals than those by EM particles due to the longer trajectories in water. In the MILAGRO experiment, a so-called muonlayer of PMTs was deployed at $7 \mathrm{~m}$ beneath the water surface also in a $3 \mathrm{~m} \times 3 \mathrm{~m}$ grid to detect those muon signals. The MILAGRO experiment was a successful attempt at using EAS detection technique in terms of angular resolution of the shower direction, $0.4^{\circ}$, and the $\gamma / \mathrm{CR}$ discrimination capability. These are two key parameters in order to enhance the sensitivity to the $\gamma$-ray sources. Their improvements both cause the reduction of the denominator, $\sqrt{N_{C R}}$, in the signal-to-noise ratio. It was remarkable that the $4800 \mathrm{~m}^{2}$ detector achieved a sensitivity of 1 CU even at the altitude of $2600 \mathrm{~m}$ a.s.1., where EASs of energies around $10 \mathrm{TeV}$ had severely decayed in atmosphere. In this regard, the water Cherenkov technique performed better than scintillator because all EAS particles were detected, including secondary $\gamma$-rays which cannot be detected by thin scintillator plates. Because photons are about a factor of 10 more than electrons and positrons in a shower, the efficient detection of those photons in water enables acceptable measurements of the EAS's fronts even at the late stages of shower development. Simultaneously, it demonstrated that the muon content in an EAS is a powerful discrimination tool in $\gamma / C R$ separation, even if the exact number of muons in an EAS is not necessarily precisely measured.

As a pioneer WCD experiment, MILAGRO provided us many good lessons at technical level as well. For instance, the importance of avoiding the optical cross-talking between photo-sensors is a good example to improve the angular resolution. Another very useful lesson is that the muon content could be sufficiently well measured without the bottom layer of PMTs in the purpose of $\gamma / C R$ separation. The size of the pond is crucial as well, it has to be big so that the entire shower front can be sufficiently contained. At relatively low altitude, shower fluctuation eventually destroys the shower energy measurement. However, the last two points are common knowledge, not to specifically apply to the WCD technique. 
Most of those improvements were justified in a better designed and realized experiment HAWC [8], located at Sierra Negra, Mexico $\left(18^{\circ} 59^{\prime} 41^{\prime \prime} \mathrm{N} 97^{\circ} 18^{\prime} 30^{\prime \prime} \mathrm{W}\right)$ at an altitude of $4100 \mathrm{~m}$ a.s.l. with an effective size of $22,500 \mathrm{~m}^{2}$. The sensitivity of $0.1 \mathrm{CU}$ for $\gamma$-ray detection was achieved with an angular resolution of $\sim 0.2^{\circ}$ at few $\mathrm{TeV}$ and even close to $0.1^{\circ}$ around $10 \mathrm{TeV}$ for well selected events. Thanks to the large size and high altitude, HAWC also achieved a good shower energy resolution of better than $20 \%$. Many discoveries have been made since it was built in 2015, including the pulsar halos associated with Geminga and Monogem [9].

The $\mathrm{AS}_{\gamma}$ experiment deployed muon detector over a large area of $3600 \mathrm{~m}^{2}$ about 23 years after its phase-I operation. The $\gamma / C R$ discrimination power was significantly improved with the muon content being well measured for showers falling inside the surface array. This boosted the sensitivity of the array to $\sim 0.1 \mathrm{CU}$ at higher energy than HAWC, $\sim 20 \mathrm{TeV}$. This enabled the detection of the most energetic photon at $\sim 450 \mathrm{TeV}$ from the Crab Nebula at the time of 2019 [10].

\section{Low and High Threshold Energy and Field of View of $\gamma$-ray Detection}

By 2006, the ARGO-YBJ experiment deployed $5600 \mathrm{~m}^{2}$ RPCs in a warehouse at Yangbajing, China, at $4300 \mathrm{~m}$ a.s.l. $\left(30^{\circ} 06^{\prime} 38^{\prime \prime} \mathrm{N} 90^{\circ} 31^{\prime} 50^{\prime \prime} \mathrm{E}\right)$. With an active coverage area of 93\%, most secondary charged particles in an EAS event below few $\mathrm{TeV}$ were recorded, as shown in Figure 2. At such altitude, this allowed a detection of showers at energies as low as $300 \mathrm{GeV}$. The shower arrival directions were measured with a resolution of $1.0^{\circ}$ around $1 \mathrm{TeV}$. A sensitivity of $0.7 \mathrm{CU}$ was achieved without any $\gamma / \mathrm{CR}$ discrimination capability.
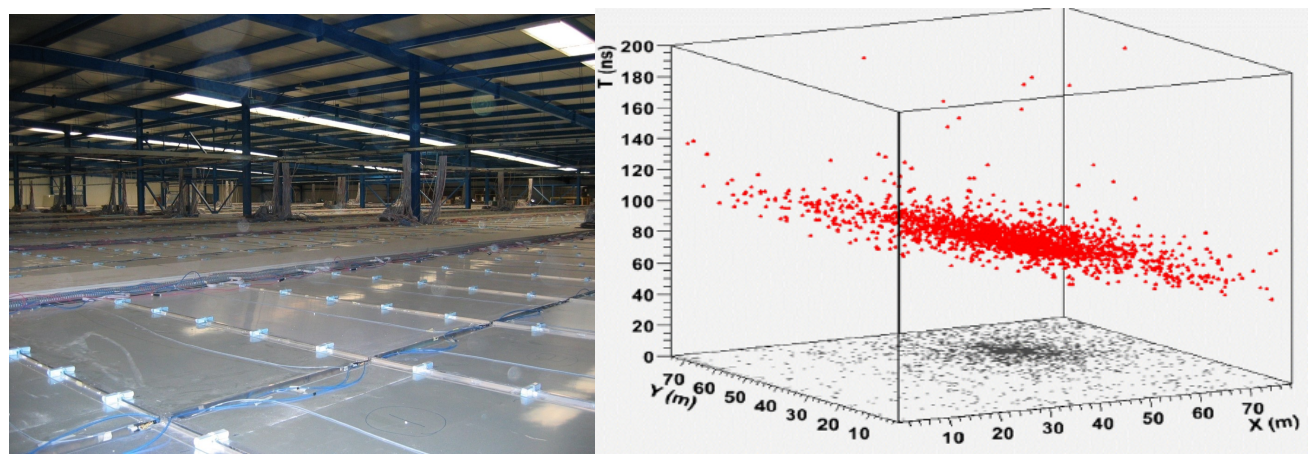

Figure 2. ARGO-YBJ detectors and an example of recoded event. In the left panel, the inside view of the ARGO-YBJ hall with RPCs deployed on the floor. Each RPC has a size of $3.3 \mathrm{~m}^{2}$ including 10 units, each of which measures number of particles up to 8 and times the arrival of those particles with a resolution of $\sim 1 \mathrm{~ns}$. In the right panel, an event recorded by the center array. The vertical axis is the timing of the particles in each unit whose location is shown in the $x-y$-plane of the array.

With the low energy threshold, the median EAS energy for the lowest bin of number of hits measured in a shower (i.e., 20-39) reached $300 \mathrm{GeV}$. ARGO-YBJ opened a window to monitor the transient phenomena in a range of $150 \mathrm{Mpc}$ using such a round-the-clock operated wide field-of-view (FoV) detector. Flares of Mrk421 and Mrk501 had been studied in detail. A hardening of their Inverse Compton (IC) spectra during the flares was evidently observed [11] with a strong correlation to the similar features of the synchrotron spectra observed by X-ray telescopes. Unfortunately, no Gamma Ray Burst (GRB) was detected due to the threshold, which was not sufficiently low, before the operation stopped in 2013.

The wide FoV revealed interesting features of galactic $\gamma$-ray sources, including the identification of Cygnus Cocoon in the TeV band [12]. A couple of 'point-sources' were found to be spatially extended. The integrated $\gamma$-ray fluxes over the extended regions were found higher than 'point-sources' observed by using imaging air Cherenkov telescopes (IACT) instruments. J1908+0621 was a typical example [13]. Matter distributions around sources, such as molecular clouds, should be considered for investigations of those extended sources. They could be the targets hit by the primary CRs accelerated in PeVatrons 
and emit very energetic $\gamma$-rays through $\pi^{0}$ decays. These extended systems may bring us extra information about $\gamma$-ray radiation mechanism. As electrons typically do not reach distant clouds due to short cooling distance, this may provide further constraints on the origin of the $\gamma$-rays.

Many lessons have been learned about utilizing the EAS technique for $\gamma$-ray detection. There is a clear low energy limit of around $100 \mathrm{GeV}$ between $4000 \mathrm{~m}$ to $5000 \mathrm{~m}$ a.s.l. Shower fluctuation limits angular resolution. A lack of $\gamma$-ray specific signature in its EAS versus the CR showers limits the sensitivity of $\gamma$-ray detection. In the EAS detection, secondary $\gamma$-rays are an important component that should not be ignored. WCD technique that fully uses all $\gamma$-rays and $e^{+}, e^{-}$in shower front achieves better angular resolution at same energy than the RPC technique. Moreover, the outstandingly big Cherenkov light signals generated by muons on top of the shower front could be used to differentiate CR showers from $\gamma$-ray showers. However, CR showers under several hundred GeVs do not have sufficient muon content to be detected as an indicator. To carry out precise spectral and morphologic measurements for $\gamma$-ray sources, experiments should go into the high energy domain to maximize the advantages of EAS technique. In the low energy domain, i.e., below several hundred GeV, EAS techniques can take advantage of the wide FoV and 24-h operation to monitor all kinds of transient phenomena. Those observations have more weight in the era of multi-messenger astronomy.

\section{LHAASO: The New Generation of the $\gamma$-ray Experiment}

The Large High Altitude Air Shower Observatory (LHAASO) [14] [15] is a complex of EAS detector arrays, located at Mt. Haizi, China $\left(29^{\circ} 21^{\prime} 27.6^{\prime \prime} \mathrm{N}, 100^{\circ} 08^{\prime} 19.6^{\prime \prime} \mathrm{E}\right)$ at $4410 \mathrm{~m}$ a.s.l. The site is at the edge of the Qingzang plateau near Daocheng, Sichuan Province. LHAASO consists of uniformly distributed EAS arrays covering an area of $1.3 \mathrm{~km}^{2}$ (KM2A) with 5216 scintillator counters (ED, $1 \mathrm{~m}^{2}$ active area) and 1188 muon detectors (MD, a WCD of $36 \mathrm{~m}^{2}$ and $1.2 \mathrm{~m}$ depth, buried under $2.5 \mathrm{~m}$ of ground). EDs are spaced $15 \mathrm{~m}$ apart and MDs at $30 \mathrm{~m}$. At the center of the array, the WCD Array (WCDA) covers $78,000 \mathrm{~m}^{2}$. The Wide FoV air Cherenkov / fluorescence Telescope Array (WFCTA) of 18 telescopes is also deployed. With the exception of the WFCTA, designed for charged CR detection and knees of the spectra of individual CR species, the main deployment of LHAASO detectors are for $\gamma$-ray astronomical observation. The layout of the entire LHAASO array is illustrated in Figure 3.

WCDA at the center of the LHAASO array is designed for surveying of VHE $\gamma$ ray sources in the northern hemisphere using the WCD technique. 3120 detector units, $5 \mathrm{~m} \times 5 \mathrm{~m}$ each and optically separated with black curtains, are equipped with a pair of PMTs, bigger and smaller, at $4 \mathrm{~m}$ beneath the water surface to measure the arrival time and amount of Cherenkov light generated by EAS particles in the shower front, respectively. Such a layout enables an angular resolution of $0.5^{\circ}$ at the most favorable energy of $2 \mathrm{TeV} .99 .5 \%$ of $\mathrm{CR}$ showers can be rejected as background using the outstanding signals generated by muons at certain distance from the shower cores. A sensitivity of $1 \% \mathrm{CU}$ is achieved for the all-sky survey. In 2/3 of the units, the larger PMTs have 20" photocathodes so that WCDA still have a sensitivity of $0.5 \mathrm{CU}$ at $70 \mathrm{GeV}$. This enables the WCDA to be an efficient monitor for transient phenomena, such as GRBs, in an 1/7 of the northern sky at any moment. A dynamic range from 1 to 3000 photoelectrons (PE) of a single unit allows a good measurement of the spectral energy distribution (SED) of a $\gamma$-ray source from 0.5 to $10 \mathrm{TeV}$. 


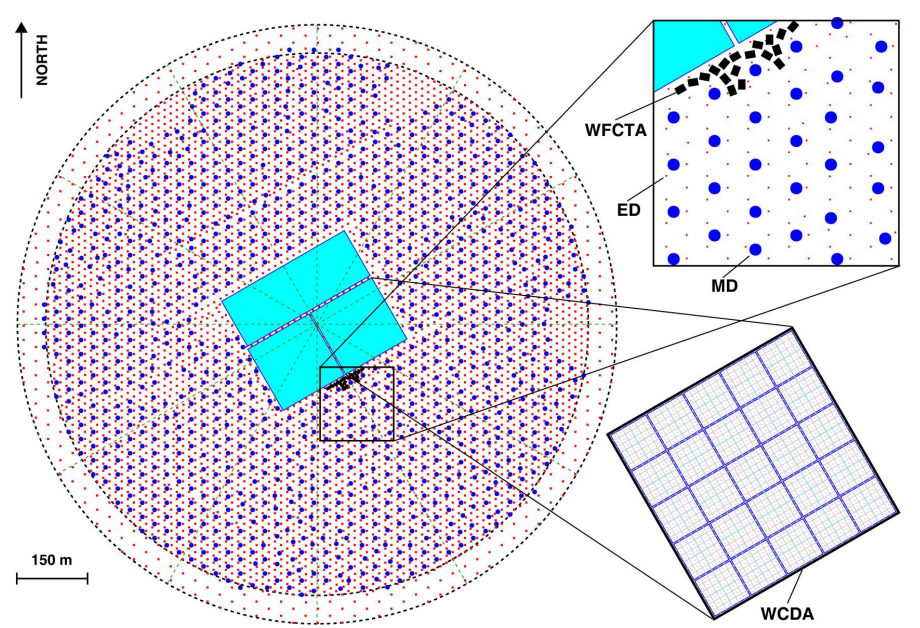

Figure 3. Schematic of LHAASO layout. Red dots represent 5216 EDs distributed in the inner circular array of $1 \mathrm{~km}^{2}$ with the spacing of $15 \mathrm{~m}$, and in the outer ring with the spacing of $30 \mathrm{~m}$. The sub-array in the ring is for vetoing showers which have cores outside the $1 \mathrm{~km}^{2}$ array. Blue filled circles represent $1188 \mathrm{MDs}$ in the $1 \mathrm{~km}^{2}$ array with the spacing of $30 \mathrm{~m}$. Light blue rectangles at the center of the site and enlarged represent WCDA in three ponds. Enlarged pond has 900 units that are divided into 25 clusters and covering an area of $150 \mathrm{~m} \times 150 \mathrm{~m}$. Black rectangles near the WCDA represent 18 telescopes in WFCTA. In the enlarged part, the detailed locations and orientations of the telescopes are indicated as well.

KM2A covers the higher energy range from $10 \mathrm{TeV}$ to few $\mathrm{PeV}$ with the sensitivity of also $1 \% \mathrm{CU}$ at the favorable energy around $50 \mathrm{TeV}$ for $\gamma$-ray detection. This means that a source with a flux as faint as $2 \times 10^{-14} \mathrm{TeV}^{-2} \mathrm{~s}^{-1}$ above $100 \mathrm{TeV}$ can be detected in one year with a significance of $5 \sigma$. Such a capability is mainly due to two features of KM2A. First, the active area of MDs reaches $4 \%$ of the total area of the entire $1 \mathrm{~km}^{2}$ array. This enables a rejection rate of CR background of $10^{-4}$ at $100 \mathrm{TeV}$ and $10^{-5}$ above $600 \mathrm{TeV}$, meaning essentially a 'background free' detection for $\gamma$-ray sources above few hundred $\mathrm{TeV}$, in other name 'PeVatrons'. Secondly, the angular resolution of $0.3^{\circ}$ for the shower arrival direction using the array of EDs with a spacing of $15 \mathrm{~m}$ and a clock synchronization accuracy of $0.5 \mathrm{~ns}$ [16] for all 5216 EDs. Further contributing to the $0.3^{\circ}$ resolution, a lead plate of $5 \mathrm{~mm}$ was installed on top of each scintillator in ED to convert EAS secondary $\gamma$ rays into pairs of $e^{+}$and $e^{-}$.

LHAASO is, therefore, a dedicated facility of search for PeVatrons via UHE $\gamma$-rays with unprecedented sensitivity. This is no doubt the most promising step of unveiling the UHE CR origins. Before the entire array was deployed in July, 2021, the 1/2 KM2A started operation at the beginning of 2020 and followed by another $1 / 4$ of the array merged in the data stream by the end of the same year. By March 2021, 530 UHE photons had been collected from 12 sources with almost no CR background. This reveals the PeVatrons in our galaxy unambiguously. The significance threshold had been set as $7 \sigma$ in the PeVatron search. Except for very few newly discovered PeVatrons, most of the PeVatrons have been well-known and found in the VHE $\gamma$-ray catalog. Our galaxy is actually full of PeVatrons, almost evenly distributed in the disk. Most of the spectra of the UHE photons do not have clear 'cut-off' feature. This indicates that the PeVatrons may be accelerating particles to even higher energies than what have been reported in Ref. [17] as the maximal energies of photons ever detected. In fact, two super-PeV photons have been detected from the Crab at 1.1 PeV and the Cygnus region at $1.4 \mathrm{PeV}[17,18]$. This indicates the existence of super-PeVatrons in our galaxy. The super-PeVatrons, certainly not limited to those two, may be responsible for the flux of CRs above the knees. These first discoveries mark the onset of UHE $\gamma$-ray astronomy. In the following years, more PeVatrons will be unveiled and their detailed radiation features will be discovered, thus bringing us closer to the goal of finding the origins of CRs. 
As one of the PeV $\gamma$-ray sources, the Crab Nebula has been measured in detail over 3.5 orders of magnitude of energy from $0.5 \mathrm{TeV}$ to $1.1 \mathrm{PeV}$ using LHAASO. WCDA measured the SED in the energy range from 0.5 to $12 \mathrm{TeV}$ and KM2A measured the energy range from $12 \mathrm{TeV}$ and above. These two components of the LHAASO detector provide independent overlapping measurements of the $\gamma$-ray flux of the 'standard candle' at the same energy bin at $12 \mathrm{TeV}$, thus providing cross-checking inside a single collaboration. The agreement of the SED measurement with other experiments below $100 \mathrm{TeV}$ affirms the maturity of the techniques [18]. Adding the measurements in the highest energy band to the multiwavelength analysis, many interesting features of the Crab Nebula are unveiled. For such a well-known point-source, LHAASO data supports the well-established radiation mechanism at least up to $100 \mathrm{TeV}$, i.e., strong pulsar wind of $e^{+} e^{-}$forms the nebula with termination shocks that further accelerate $e^{+} e^{-}$to very high energies. It is found that a simple one-zone model is able to produce the very complex SED over 10 decades of energy. In this model, a same bulk of $e^{+}$and $e^{-}$are assumed to make the synchrotron radiation in a magnetic field of $112 \mu \mathrm{G}$ and inverse-Compton scattering on soft photon fields, e.g., CMB photons, simultaneously. The agreement between data and the simple model is impressive. However, we do observe some deviations of LHAASO data from such a beautiful model above $50 \mathrm{TeV}$ at a level of $4 \sigma$ [18]. This makes the origin of the highest energy photons unclear, either from electrons or possibly protons. Both indications imply important outcomes which will be clarified in 2 or 3 years. According to what we have learned from the observations using 3/4 of the LHAASO array, 1 or 2 photons at energies around $1 \mathrm{PeV}$ are expected to be detected per year with the full scale array. More importantly, the SED above $50 \mathrm{TeV}$ will be measured much more precisely while we are waiting for the anticipated $\mathrm{PeV}$ photons.

\section{Summary}

In this paper, the development of the EAS particle detection arrays as $\gamma$-ray astronomical instruments over the past 30 years has been summarized. The performances of the instruments can be summarized in a plot of the integrated sensitivities of their $\gamma$-ray detection as a function of $\gamma$-ray energy, as shown in Figure 4 . Three generations of the detectors using various techniques are clearly shown in the figure according to the improvement of their sensitivity from $1 \mathrm{CU}$ for the 1st generation to $0.01 \mathrm{CU}$ for the 3rd generation represented by LHAASO. It is quite clear that the WCD technique and scintillator detector are very useful, especially in taking full advantage of high altitude sites. We have also realized that the high altitudes are important. Since photons will be absorbed even inside our galaxy at energies higher than few PeV, there is no need to go higher than $4500 \mathrm{~m}$ a.s.l. where PeV EAS may have already reached the ground before showers developed to their maxima. After exploring for 30 years, EAS techniques are finally found essential in the suitable energy range above few tens of $\mathrm{TeV}$ when PeVatrons are suddenly found everywhere in our Milky Way. Soon, we will witness a wave of discoveries in finding the acceleration and radiation mechanisms behind the PeVatrons or even super-PeVatrons. The very preliminary investigation of the Crab Nebula using LHAASO, as the first attempt, has already shown many unknown fascinating features of this very familiar celestial body. In a couple more years of data collection, the production of photons above $1 \mathrm{PeV}$ may be clarified with surprising discoveries. This is just the first observations in the UHE domain of $\gamma$-ray astronomy. Many more investigations of PeVatrons, discovered and to be discovered, are anticipated. It is doubtless that another milestone has been passed in the way of finding the origins of high energy CRs. 


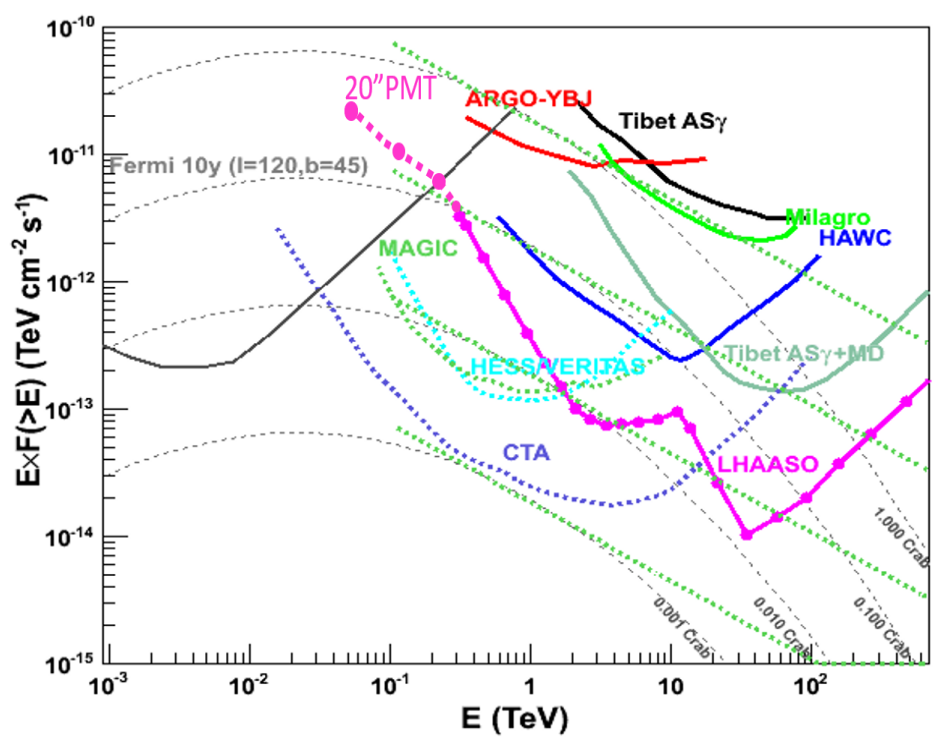

Figure 4. Sensitivities of VHE and UHG $\gamma$-ray astronomical instruments as functions of $\gamma$-ray energy, E. The Crab Nebula SED in a log-parabola functional form in gray short dashed lines has been adopted from Ref. [15], and the power law extension has been normalized at $1 \mathrm{TeV}$ to be the same as the log-parabola. The ground based EAS experiments, $\mathrm{AS}_{\gamma}+\mathrm{MD}$ [19], HAWC [20], ARGO-YBJ [21] and MILAGRO [22] are represented by colored solid lines. The IACT experiments, CTA [23], VERITAS [24], HESS [25],MAGIC [26] are represented by colored dotted lines. The 10 year sensitivity of FERMI-LAT [27] is represented by the gray solid line. LHAASO sensitivity represented by the pink solid line is from Ref. [15] and the extension in low energies down to $50 \mathrm{GeV}$ in dashed line is newly estimated based on the upgraded deployment using 20" PMTs.

Funding: This research work was funded by the Chengdu Management Committee of Tianfu New Area. It was also supported by following grants. The National Key R\&D program of China under the grant 2018YFA0404201, 2018YFA0404202 and 2018YFA0404203.

Institutional Review Board Statement: Not applicable.

Informed Consent Statement: Not applicable.

Data Availability Statement: No data were used in this reviewing paper.

Acknowledgments: The author would like to thank all the supports from colleagues and friends in various experiments that I participated, namely AS $\gamma$, CASA-MIA, ARGO-YBJ and LHAASO. The author also appreciate the proof reading and efforts in improving English presentation of the MS by Andrew J Cao.

Conflicts of Interest: The author declare no conflict of interest.

\section{References}

1. Samorski, M.; Stamm, W. Detection of 2X10(15) to 2X10(16)EV Gamma-Rays From Cygnus-X-3. Astrophys. J. 1983, 268, 17. [CrossRef]

2. Borione, A.; Covault, C.E.; Cronin, J.W.; Fick, B.E.; Gibbs, K.G.; Krimm, H.A.; Mascarenhas, N.C.; Mackay, T.A.; Muller, D.; Newport, B.J.; et al. A Large Air Shower Array to Search for Astrophysical Sources Emitting Gamma-Rays With Energies Greater-Than-Or-Equal-To-10(14) EV. Nucl. Inst. Methods Phys. Res. A 1994, 346, 329-352. [CrossRef]

3. Amenomori, M.; Cao, Z.; Ding, L.K.; Feng, Z.Y.; Hibino, K.; Hotta, N.; Huang, Q.; Huo, A.X.; Jia, H.Y.; Jiang, G.Z.; et al. Search for Steady Emission of 10-Tev Gamma-Rays from the Crab-Nebula, Cygnus-X-3, and Hercules-X-1 Using the Tibet Air Shower Array. Phys. Rev. Lett. 1992, 69, 2468. [CrossRef] [PubMed]

4. Weekes, T.C.; Cawley, M.F.; Fegan, D.J.; Gibbs, K.G.; Hillas, A.M.; Kwok, P.W.; Lamb, R.C.; Lewis, D.A.; Macomb, D.; Porter, N.A.; et al. Observation of Tev Gamma-Rays from the Crab-Nebula Using the Atmospheric Cerenkov Imaging Technique. Astrophys. J. 1989, 342, 379-395. [CrossRef] 
5. Borione, A.; Chantell, M.C.; Covault, C.E.; Cronin, J.W.; Fick, B.E.; Fowler, J.W.; Fortson, L.F.; Gibbs, K.G.; Green, K.D.; Newport, B.J.; et al. High Statistics Search for Ultrahigh Energy Gamma-Ray Emission from Cygnus X-3 and Hercules X-1. Phys. Rev. D 1997, 55, 1714-1731. [CrossRef]

6. Borione, A.; Catanese, M.A.; Chantell, M.C.; Covault, C.E.; Cronin, J.W.; Fick, B.E.; Fortson, L.F.; Fowler, J.F.; Gibbs, K.G.; Glasmacher, M.A.K.; et al. A Search for Ultra-High-Energy Gamma-Ray Emission from the Crab Nebula and Pulsar. Astrophys. J. 1997, 481, 313-326. [CrossRef]

7. Yodh, G.B. The Milagro Gamma Ray Observatory. Nucl. Phys. B 1997, 52, 264-268. [CrossRef]

8. Abeysekar, A.U.; Albert, A.; Alfaro, R.; Alvarez, C.; Alvarez, J.D.; Camacho, J.R.; Arceo, R.; Arteaga-Velazquez, J.C.; Arunbabu, K.P.; Rojas, D.; et al. Measurement of the Crab Nebula Spectrum past $100 \mathrm{TeV}$ with Hawc. Astrophys. J. 2019, 881, 134.

9. Abeysekara, A.U.; Albert, A.; Alfaro, R.; Alvarez, C.; Alvarez, J.D.; Arceo, R.; Arteaga-Velazquez, J.C.; Rojas, D.A.; Solares, H.A.A.; Barber, A.S.; et al. Extended Gamma-Ray Sources around Pulsars Constrain the Origin of the Positron Flux at Earth. Science 2017, 358, 911-914. [CrossRef] [PubMed]

10. Amenomori, M.; Bao, Y.W.; Bi, X.J.; Chen, D.; Chen, T.L.; Chen, W.Y.; Chen, X.; Chen, Y.; Cui, S.W.; Ding, L.K.; et al. First Detection of Photons with Energy beyond $100 \mathrm{TeV}$ from an Astrophysical Source. Phys. Rev. Lett. 2019, 123, 051101.

11. Bartoli, B.; Bernardini, P.; Bi, X.J.; Bleve, C.; Bolognino, I.; Branchini, P.; Budano, A.; Melcarne, A.K.C.; Camarri, P.; Cao, Z.; et al. Long-Term Monitoring of Mrk 501 for Its Very High Energy Gamma Emission and a Flare in 2011 October. Astrophys. J. 2012, 758, 2. [CrossRef]

12. Bartoli, B.; Bernardini, P.; Bi, X.J.; Branchini, P.; Budano, A.; Camarri, P.; Cao, Z.; Cardarelli, R.; Catalanotti, S.; Chen, S.Z.; et al. Identification of the TeV Gamma-Ray Source Argo J2031+4157 with the Cygnus Cocoon. Astrophys. J. 2014, 790, 152. [CrossRef]

13. Bartoli, B.; Bernardini, P.; Bi, X.J.; Bleve, C.; Bolognino, I.; Branchini, P.; Budano, A.; Melcarne, A.K.C.; Camarri, P.; Cao, Z.; et al. Observation of the TeV Gamma-Ray Source Mgro J1908+06 with ARGO-YBJ. Astrophys. J. 2012, 760, 110. [CrossRef]

14. Cao, Z. A Future Project at Tibet: The Large High Altitude Air Shower Observatory (Lhaaso). Chin. Phys. C $2010,34,249-252$.

15. He, H. For the LHAASO Collaboration. Radiat. Detect. Technol. Methods 2018, 2, 7. [CrossRef]

16. Gong, G.; Chen, S.; Du, Q.; Li, J.; Liu, Y.; He, H. Sub-nanosecond Timing System Design and Development for LHAASO Project. In Proceedings of the ICALEPCS 2011, Grenoble, France, 10-14 October 2011.

17. Cao, Z.; Aharonian, F.A.; An, Q.; Axikegu; Bai, L.X.; Bai, Y.X.; Bao, Y.W.; Bastieri, D.; Bi, X.J.; Bi, Y.J.; et al. LHAASO Collaboration. Ultrahigh-Energy Photons Up to 1.4 Petaelectronvolts from 12 Gamma-Ray Galactic Sources. Nature 2021, 594, 33-36. [CrossRef]

18. Cao, Z.; Aharonian, F.; An, Q.; Axikegu; Bai, L.X.; Bai, Y.X.; Bao, Y.W.; Bastieri, D.; Bi, X.J.; Bi, Y.J.; et al. LHAASO Collaboration. PETA-Electron Volt Gamma-Ray Emission from the Crab Nebula. Science 2021, 373, 425-430. [PubMed]

19. Huang, J. Recent Results and the Next Step of the Tibet AS $\gamma$ Experiment. In Proceedings of the 32th ICRC, Beijing, China, 11-18 August 2011; Volume 12, pp. 107-118.

20. Abeysekara, A.U.; Alfaro, R.; Alvarez, C.; Alvarez, J.D.; Arceo, R.; Arteaga-Velazquez, J.C.; Solares, H.A.A.; Barber, A.S.; Baughman, B.M.; Bautista-Elivar, N.; et al. HAWC Collaboration. Sensitivity of the High Altitude Water Cherenkov Detector to Sources of Multi-TeV Gamma Rays. Astropart. Phys. 2013, 50-52, 26-32. [CrossRef]

21. Bartoli, B.; Bernardini, P.; Bi, X.J.; Bolognino, I.; Branchini, P.; Budano, A.; Melcarne, A.K.C.; Camarri, P.; Cao, Z.; Cardarelli, R.; et al. ARGO-YBJ Collaboration. TeV Gamma-Ray Survey of the Northern Sky Using the ARGO-YBJ Detector. Astrophys. J. 2013, 779, 27. [CrossRef]

22. DeYong, T.; for HAWC Collaboration. The HAWC Observatory. NIM A 2012, 692, 72-76. [CrossRef]

23. Available online: https://www.cta-observatory.org/science/ctao-performance/ (accessed on 8 September 2021).

24. Available online: https://veritas.sao.arizona.edu/about-veritas/veritas-specifications (accessed on 8 September 2021).

25. Holler, M.; et al. HESS Collaboration. Photon Reconstruction for H.E.S.S. Using a Semi-Analytical Model. PoS ICRC2015 2015, 34,980

26. Aleksić, J.; Ansoldi, S.; Antonelli, L.A.; Antoranz, P.; Babic, A.; Bangale, P.; Barcelo, M.; Barrio, J.A.; Gonzalez, J.B.; Bednarek, W.; et al. MAGIC Collaboration. The Major Upgrade of the Magic Telescopes, Part II: A Performance Study Using Observations of the Crab Nebula. Astropart. Phys. 2016, 72, 76-94. [CrossRef]

27. Available online: http://www.slac.stanford.edu/exp/glast/groups/canda/lat_Performance.htm (accessed on 8 September 2021). 